\title{
The global response to the COVID-19 pandemic: how have immunology societies contributed?
}

Faith Osier, Jenny P. Y. Ting $\mathbb{D}^{-}$, John Fraser, Bart N. Lambrecht, Marta Romano, Ricardo T. Gazzinelli, Karina R. Bortoluci(D, Dario S. Zamboni(D, Arne N. Akbar $(\mathbb{D}$, Jennie Evans, Doug E. Brown, Kamala D. Patel, Yuzhang Wu, Ana B. Perez, Oliver Pérez, Thomas Kamradt, Christine Falk, Mira Barda-Saad, Amiram Ariel(D), Angela Santoni, Francesco Annunziato, Marco A. Cassatella, Hiroshi Kiyono $\mathbb{D}$, Valeriy Chereshnev, Alioune Dieye, Moustapha Mbow, Babacar Mbengue, Maguette D. S. Niang (1) and Melinda Suchard (D)

Abstract |The COVID-19 pandemic is shining a spotlight on the field of immunology like never before. To appreciate the diverse ways in which immunologists have contributed, Nature Reviews Immunology invited the president of the International Union of Immunological Societies and the presidents of 15 other national immunology societies to discuss how they and their members responded following the emergence of severe acute respiratory syndrome coronavirus 2 (SARS-CoV-2).

\section{Can you highlight some of the ways in which your immunology society and its members have contributed to the response to the COVID-19 pandemic?}

International Union of Immunological Societies: Faith Osier. Unprecedented times call for unprecedented actions. Immunologists and vaccine developers across the world have committed to producing vaccines against COVID-19 in timelines previously considered unthinkable. Immunologists globally have partnered and engaged with epidemiologists, clinicians, public health officials, regulatory agencies and funding bodies to fast-track research to better understand the disease. On 11 March 2020, the World Health Organization declared COVID-19 a pandemic: within weeks, a flurry of research activity was already under way. The scientific literature was flooded with studies describing the disease (epidemiology, transmission, onset and pathogenesis) as well as prognostic biomarkers. Concurrently, innovative study designs facilitated rapid collection of data on potential immune-based therapies and drugs in multiple sites simultaneously. Old and new potential therapies were equally put to the test. And we already have some answers.

Education and communication between scientists across disciplines, and between scientists and the general public, has flourished. Enabled by contemporary interactive online platforms, immunologists took an early lead in organizing scientific webinars for information sharing and collaborations and to spur cross-disciplinary research. The International Union of Immunological Societies (IUIS) brought together more than 6,000 immunologists from more than 100 countries in a series of weekly COVID-19 webinars ${ }^{1}$. These not only spanned the breadth of immunology but also reflected on our responsibility to maintain the integrity of rigorous scientific peer review, especially during a pandemic. Our speakers reflected our rich diversity and expertise across regional and national boundaries, and smashed stereotypes with regard to gender, race and age. We contributed to our local, national and regional response strategies. Like never before, we engaged vigorously with the public in our respective constituencies and mustered the composure to face our own vulnerability. Although we still do not have all the answers, together our immunology societies have been an incredible global resource, as is highlighted in the contributions herein and in FIG. 1. (Editor note: see FIG. 2 for biographies of the presidents of the immunology societies featured in this article and Supplementary information for biographies of all the contributors.)

American Association of Immunologists: Jenny P. Y. Ting. The pandemic immediately forced the American Association of Immunologists (AAI) to cancel our annual meeting, IMMUNOLOGY2020, and to work remotely. AAI members are working at the forefront of the pandemic, conducting critical immunological research that will support clinical solutions. US laboratories, however, have been challenged by pandemic restrictions, and our members need more support than ever. To provide this, the AAI developed a three-pronged approach prioritizing (1) advocacy, (2) dissemination of information and (3) continued funding.

AAI public affairs activities were critical components of the advocacy plan ${ }^{2}$. Since March 2020, AAI members have submitted testimonies, issued statements, written and co-signed letters, and advocated additional funding. Efforts included writing to President Donald Trump and Congressional leaders urging that they heed the advice of scientific/public health leaders, including AAI member Anthony Fauci ${ }^{3}$; writing to National Institutes of Health (NIH) Director Francis Collins requesting justification for terminating an NIH-funded grant focusing on understanding the risk of bat coronavirus emergence ${ }^{4}$; advocating supplemental funding for federal science agencies, including the NIH, for pandemic-related research losses and additional trainee support; and issuing a statement opposing actions taken by the Trump administration that will damage international scientific collaboration ${ }^{5}$.

AAI journals accelerated the time taken for reviews of COVID-19 papers to 1 week and made all coronavirus-related publications free online to speed up the dissemination of crucial information ${ }^{6}$. Due to laboratory closures, authors and reviewers were given more flexibility? 
The AAI sustained education and funding initiatives. Although the IMMUNOLOGY2020 meeting was cancelled, the AAI published all submitted abstracts free online ${ }^{8}$. Furthermore, it established a 'COVID-19 Resources and Information Center", transitioned AAI-led immunology courses from onsite to virtual ${ }^{10}$ and continued funding for all AAI Fellowships despite laboratory closings $^{11}$.

Australian and New Zealand Society for Immunology: John Fraser. The Australian and New Zealand Society for Immunology is a very active and collegial society with more than 1,200 members spread across the seven states of Australia and New Zealand; and no, New Zealand is not (yet) a state of Australia. We have remained very active despite the pandemic putting a pin in all our regular events for 2020. Many of our members have talked whimsically over the years about a global pandemic and now we are living it! Immunology and infectious disease have been at the forefront of public awareness, and many of our members have been instrumental in communicating the science of immunology in lay terms to the public and, more importantly, in advising policymakers and politicians, who, I might add, have been very keen to learn some immunology. Many of our members are also part of international research teams looking for solutions to the pandemic. The Australian and New Zealand Society for Immunology is a respected scientific society within the region and has responded to the COVID-19 crisis with well-informed information and online resources such as 'Ask an Immunologist' ${ }^{\text {'2 }}$ and our 'Fireside chats' ${ }^{13}$.

Australia and New Zealand responded vigorously at the first sign of community transmission, and after enduring a brief but stringent lockdown now enjoy (almost) 'COVID-free' status in the community. However, the sudden dramatic surge in cases in the Australian state of Victoria has brought home just how easily containment control can be lost. Nevertheless, our relative success is down to two things; one, a scientifically informed and adherent public; and two, quality science-led political leadership. Attention now turns to a vaccine; which ones will work and more commonly when can we expect one. With so much anticipation, it has been difficult to caution that the massively accelerated development of so many non-optimized vaccines risks making a wrong choice. It is an important time to be an immunologist!

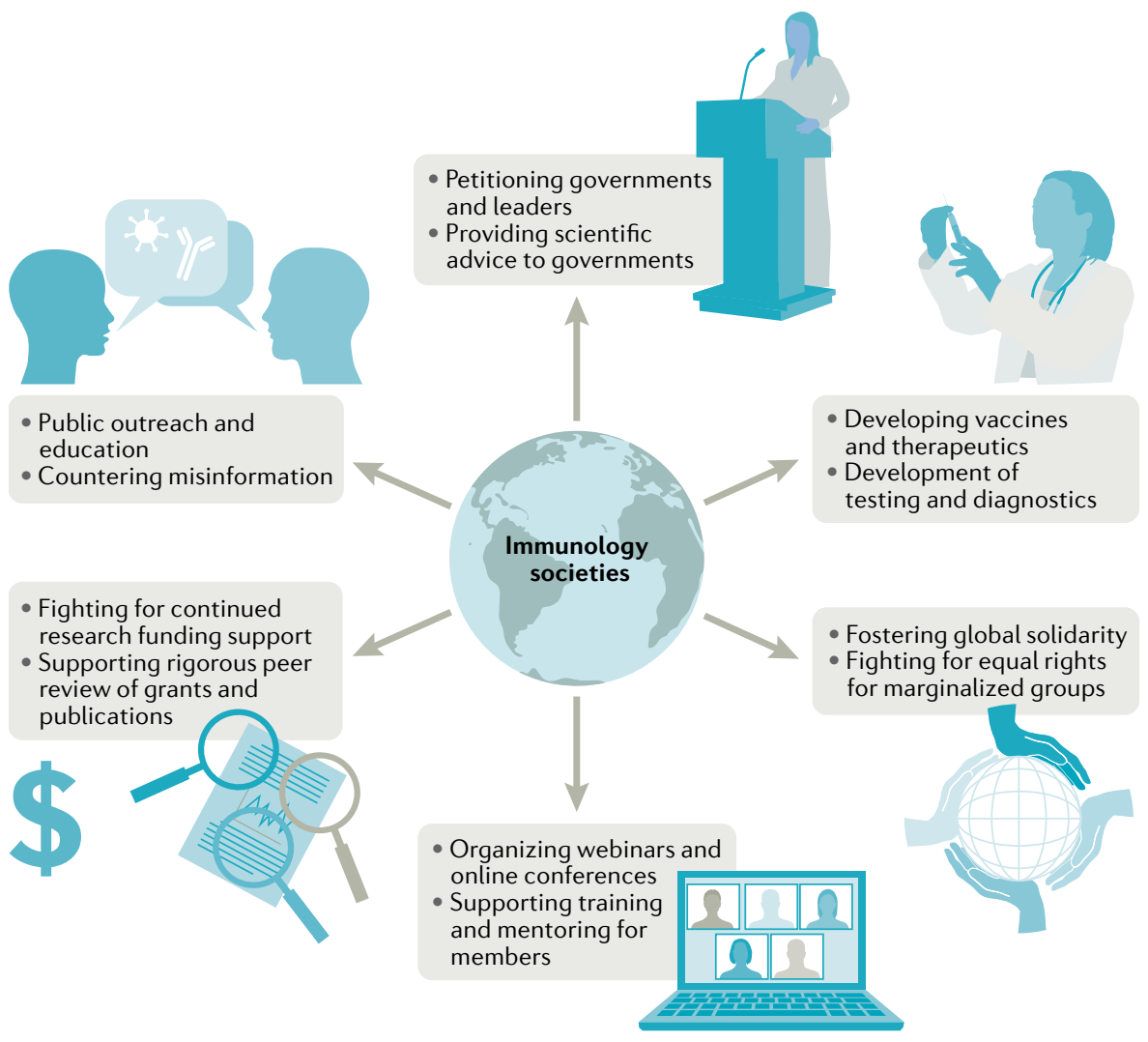

Fig. 1 Immunology societies have been vital to the global response to the COVID-19 pandemic. The diverse ways in which immunology societies have contributed during the COVID-19 pandemic are illustrated. Immunology societies have supported their members in their efforts to develop testing, therapeutics and vaccines; they have continued to fight for funding and for rigorous peer review of publications; they have organized online webinars, conferences and training programmes; and they have provided scientific advice to governments and policymakers. Immunology societies and their members have been vital for public education and for countering misinformation that has arisen during the pandemic. Finally, the immunology societies have championed equal rights and global solidarity. Recent events have drawn attention in particular to the Black lives matter movement; a number of immunology societies have published statements in support of their commitment to fostering a culture of diversity and inclusion in our immunology research communities.

Belgian Immunological Society: Bart N. Lambrecht and Marta Romano. Belgium has been hit hard by COVID-19, with close to 10,000 deaths from a population of 11.5 million. Almost $70 \%$ of deaths occurred in residential care centres. There was an early lockdown, and hospitals were well prepared to handle severe cases of COVID-19 owing to a high density of specialized intensive care unit beds in Belgium. Initially, PCR testing capacity was restricted, which led to underdiagnosis of mild cases and overestimation of deaths attributable to COVID-19, particularly in elderly people. The Belgian Immunological Society (BIS) is a small society with only 210 active members, but its members have been influential in this health crisis. Several BIS board members were part of a task force that discussed and designed all clinical interventions for COVID-19. The BIS president, a pulmonologist by training, is the principal investigator on three national trials investigating the use of recombinant granulocyte-macrophage colony-stimulating factor (ClinicalTrials. gov ID NCT04326920 $)^{14}$ anticytokine drugs targeting IL-1, IL-6 and IL-6 receptor (NCT04330638) ${ }^{15}$ and the complement C5 inhibitor zilucoplan (NCT04382755) ${ }^{16}$. These clinical trials have incorporated highly detailed immunomonitoring, including 30-colour flow cytometry, single-cell RNA sequencing and cellular indexing of transcriptomes and epitopes by sequencing (CITE-seq) analysis of more than 150 barcoded antibodies. Several BIS members are coordinating different projects, such as studies on immune correlates of protection or nationwide seroprevalence testing. Much immunological knowledge was shared during discussions with clinicians on when and how to administer convalescent plasma and how to identify 

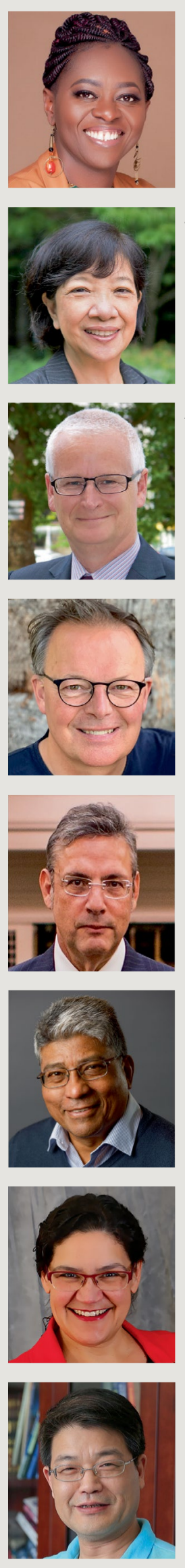

Faith Osier is Professor of Malaria Immunology and President of the International Union of Immunological Societies. She is an official \#TOGETHERBAND ambassador for the United Nations sustainable development goals and a 2018 TED Fellow.

Jenny P. Y. Ting is President of the American Association of Immunologists and Professor of Genetics, Microbiology-Immunology and the Lineberger Comprehensive Cancer Center at the University of North Carolina at Chapel Hill. She has made seminal contributions to the field of innate immunity, with a focus on the NLR family.

John Fraser is President of the Australian and New Zealand Society for Immunology and a fellow of the Royal Society of New Zealand. $\mathrm{He}$ is Professor of Molecular Medicine and Dean of the Faculty of Medical Sciences at the University of Auckland. His work focuses on antimicrobial immunity.

Bart N. Lambrecht has served as President of the Belgian Immunological Society since 2018. He is Professor of Pulmonary Medicine at Ghent University and Director of the VIB Center for Inflammation Research in Ghent. His work focuses on type 2 immunity and the lungs.

Ricardo T. Gazzinelli is President of the Brazilian Society for Immunology. He is a senior investigator at Fundação Oswaldo Cruz, Brazil, and a professor at the University of Massachusetts Medical School, USA. His research focuses on immunity to protozoan parasites.

Arne N. Akbar is Professor of Immunology at University College London and President of the British Society for Immunology. His research focuses on immunity during ageing in humans.

Kamala D. Patel is President of the Canadian Society for Immunology and a professor in the Department of Physiology and Pharmacology in the Cumming School of Medicine at the University of Calgary, Canada. Her research focuses on microscopy and leukocyte trafficking.

Yuzhang $\mathbf{W u}$ is President of the Chinese Society of Immunology and Director of the Institute of Immunology at Army Medical University, Chongqing, China.

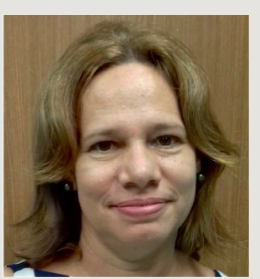

Ana B. Perez is President of the Cuban Society for Immunology. She is Head of the Cellular Immunology Laboratory in the Department of Virology in the Pedro Kourí National Institute of Tropical Medicine, Havana, Cuba. She is a professor at the Latin American University of Medical Sciences.

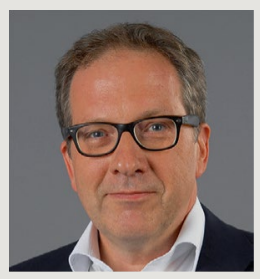

Thomas Kamradt is a medical doctor and the President of the German Society for Immunology. Since 2004 he has been Director of the Institute of Immunology of Jena University Hospital. His research focuses on T cells and chronic inflammatory diseases.
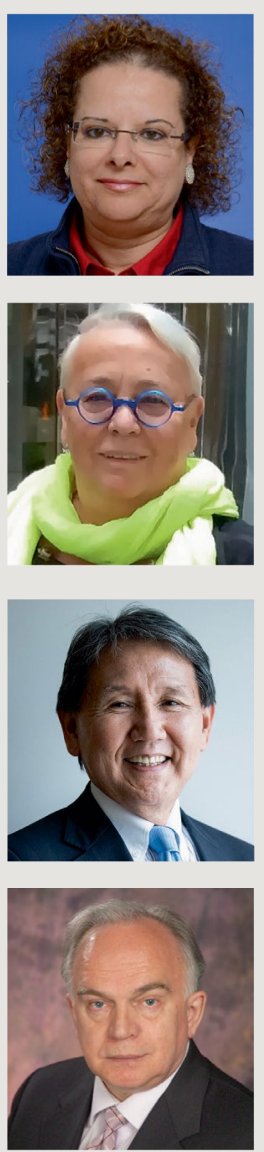

Valeriy A. Chereshnev is President of the Russian Scientific Society of Immunologists. He is a member of the Russian Academy of Sciences, a Doctor of Medicine and Scientific Director of the Institute of Immunology and Physiology. His interests include inflammation, ecological immunology and immunodeficiency.

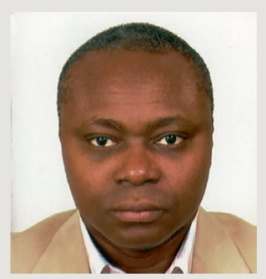

Alioune Dieye is President of the Sengalese Society of Immunology. He is Head of the Department of Immunology in the Faculty of Medicine, Pharmacy and Odontology at Cheikh Anta Diop University of Dakar. He previously served as FAIS President and as an IUIS Council member.

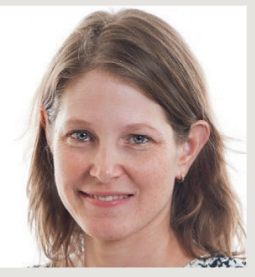

Melinda Suchard heads the Centre for Vaccines and Immunology at the National Institute for Communicable Diseases, Johannesburg. She heads the regional reference laboratories for polio, measles and rubella for the WHO and is a member of the South African National Advisory Group on Immunization. 
4 Fig. 2 | The presidents of the immunology societies. A short biography and an image of the current president of each immunology society featured in this Viewpoint are shown. FAIS, Federation of African Immunological Societies; ILC, innate lymphoid cell; IUIS, International Union of Immunological Societies; NK, natural killer; NLR, NOD-like receptor; WHO, World Health Organization. Credits: Faith Osier; Jenny P. Y. Ting, The American Association of Immunologists; John Fraser, TIGERTIGER; Bart N. Lambrecht; Ricardo T. Gazinelli; Arne N. Akbar, British Society for Immunology; Kamala D. Patel; Yuzhang Wu; Ana B. Perez; Thomas Kamradt, Jena University Hospital; Mira Barda-Saad; Angela Santoni; Hiroshi Kiyono, Japanese Society for Immunology; V. A. Chereshnev; Alioune Dieye; Melinda Suchard, KEMRI Wellcome Trust.

good donors. Discussions on the risk of antibody-mediated enhancement and cytokine storm led to alterations in trial design (NCT04429854). Finally, several BIS board members took the initiative to open up their technical platforms to increase the PCR testing capacity, which soon led to a capacity of more than 30,000 tests per day.

BIS members also advised several ministers and health-care officials on the value and significance of antibody testing, on plans for preparedness and on deciding which immunomodulatory drugs to stockpile. BIS members were frequently in the news to educate the general public on the immune response to COVID-19, the value of antibody testing and the impact of the cytokine storm on disease severity. We saw it as our duty to educate clinicians on the immunology of COVID-19 through webinars, which were very well attended. Our society also took care in identifying patients at particular risk of severe COVID-19, and all major hospitals now report their young and severely affected patients to the Belgian Primary Immune Deficiency Group, which is collecting samples and will perform in-depth genetic analyses.

\section{Brazilian Society for Immunology: Ricardo T. Gazzinelli, Karina R. Bortoluci and Dario S. Zamboni. Today, Brazil} is placed in the shamed second-highest position in terms of the number of cases of severe acute respiratory syndrome coronavirus 2 (SARS-CoV-2) infection and deaths due to COVID-19. The first official case in Brazil was recorded in February 2020. Expecting a boom in transmission, Brazilian scientists quickly mobilized to create strategies to fight SARS-CoV-2. It soon became clear that the hopes to control transmission and to treat COVID-19 would largely rely on assessment of and interventions in the immune system. The Brazilian Society for Immunology (Sociedade Brasileira de Imunologia) led the efforts to mobilize research laboratories into national and international networks to tackle COVID-19. We also intensified communications with the general media and on social networks, urging our citizens to follow scientific advice in dealing with this pandemic. This was key, as the Brazilian government minimized the importance of this disease, spreading misleading information and taking decisions not supported by high scientific standards.

Meanwhile, our scientists have been designing new vaccine strategies and trying to understand how cognate innate immune receptors for SARS-CoV-2, host cell metabolism and pro-inflammatory mediators contribute to host resistance or development of severe disease. The lack of governmental guidance makes one believe that the Brazilian president's plan is to acquire herd immunity by natural transmission, even though in many cities the occupancy of intensive care units is at its limits. Hence, Brazilian immunologists have also been preoccupied with clinical studies. The development of reliable point-of-care immunodiagnostic tests for immuno-epidemiological surveys and clinical trials involving serotherapy, immunomodulators and anti-inflammatory drugs is under way. In addition, the high rate of SARS-CoV-2 transmission and the large governmental manufacturing plants for industrial production of vaccines have made Brazil an attractive place to perform phase III clinical trials and to eventually produce the vaccines for COVID-19. A silver lining of this sad scenario has been the wider spread of scientific knowledge, in particular relating to immunology, among the general public in Brazil. In this sense, the Brazilian Society for Immunology made a key contribution with daily news, live appearances, videos and debates on our social media channels, including Facebook (@sbi.imuno, 51,306 followers), Instagram (@sbi.imuno,27,363 followers), Twitter (@sbi_imuno, 735 followers), YouTube and our society website ${ }^{17}$. In addition, many of our members have contributed to news reports in the conventional press.

British Society for Immunology: Arne N. Akbar, Jennie Evans and Doug E. Brown. The COVID-19 pandemic has seen the global immunology community come together in an unprecedented manner to drive research forwards. In the UK, the British Society for Immunology (BSI) has been at the centre of efforts and a vocal advocate for immunology in political circles and in wider public debate. For example, together with the Academy of Medical Sciences, we convened an expert taskforce on immunology and COVID-19 $\left(\mathrm{REF}^{18}{ }^{18}\right.$, which has fed into the highest levels of the UK government information on the immunology of the pandemic ${ }^{19}$. Taskforce members have also given evidence to UK parliamentary select committees on topics including immunity, vaccine development and the immunopathology of COVID-19, as well as briefing individual members of parliament.

The BSI is keen to ensure the public has access to reliable, evidence-based information on COVID-19. Through our media engagement, the voices of BSI members have featured prominently in national news reports throughout the pandemic. We also produced resources for the public to demystify the science of COVID-19 on topics including testing and vaccine development ${ }^{20}$. For our members and the wider research community, we launched our highly successful 'Connect on Coronavirus' webinar series, which sees internationally renowned speakers discuss the latest SARS-CoV-2 research ${ }^{21}$.

The BSI has made a real impact in ensuring the voice of the immunology community on COVID-19 is represented at the highest levels in the UK. We are extremely proud of what we have achieved, all made possible through our members' support. We express our deepest thanks to those who have contributed to our activities and the ongoing global COVID-19 effort.

\section{Canadian Society for Immunology:} Kamala D. Patel. The Canadian Society for Immunology (CSI) represents more than 250 biomedical researchers, clinician scientists, postdoctoral fellows and graduate students. Many are directly involved in finding solutions to the problems caused by the COVID-19 pandemic. Other members actively participate in communicating with the public to dispel the myths that surround the pandemic, serving as a source for factual information across all media platforms.

The CSI is a rapid conduit for sharing pandemic-related information with the immunology community; for example, details of webinars and other events. The CSI is also a point of contact 
for media outlets in Canada that are searching for experts to provide facts about COVID-19.

Our society actively worked to influence the Canadian Institutes of Health Research (CIHR) to reverse its cancellation of the Spring 2020 Project Grant Competition. The cancellation occurred after researchers had submitted their grants. This unprecedented response to the pandemic could have resulted in ending some research programmes as well as the loss of scientific jobs. In response to the CSI and other organizations, the CIHR re-established the competition.

Having cancelled the CSI annual conference, we took our own advice and held our annual general meeting online. This meeting was held as protests against anti-Black racism erupted in the USA, before spreading globally. Black, Indigenous and other marginalized groups face systemic racism in Canada as well. At the annual general meeting, we confronted this inequity with direct and specific actions ${ }^{22}$.

The strength of a vibrant, well-established society, such as the CSI, is that we are in a position of readiness to respond to pandemics and other crises with the power of numbers and an engaged, expert community.

Chinese Society for Immunology: Yuzhang Wu. In the very early stages of the pandemic, the Chinese Society for Immunology launched an urgent project of popularization of immunology, with the aim of helping the public to understand immunology jargon, such as 'immune defence,' 'vaccine', 'antisera and antibodies', 'herd immunity' and 'cytokine storm'. These activities helped to increase public understanding of the disease, and how they could help with maintaining immunity and self-protection. At the same time, the Chinese Society for Immunology advised the central government and local governments to rapidly start the development of immunological diagnostic, vaccine and therapeutic antibody projects. At the time of writing, more than 20 antibody test kits using colloidal gold and chemiluminescence methods for both IgM and IgG detection ${ }^{23}$ and one nucleocapsid antigen rapid detection method ${ }^{24}$ have been developed for diagnosis of COVID-19. So far, six vaccines, including four inactivated vaccines ${ }^{25}$, one adenovirus 5 -vectored vaccine ${ }^{26}$ and one mRNA-based vaccine, have been approved for clinical trials by the China Food and Drug Administration, and one of the inactivated vaccines is in a phase III clinical trial.
By use of single-cell sequencing and high-throughput screening technologies, dozens of neutralizing human monoclonal antibodies were found with preventive and therapeutic potential ${ }^{27,28}$, and one of them has been approved for clinical trials by the China Food and Drug Administration ${ }^{29}$. During the pandemic, Chinese immunologists have been involved in both basic and clinical studies of COVID-19. In collaboration with zoologists and virologists, they have developed a list of animal models, including rhesus monkeys, ACE2-transgenic mice ${ }^{30}$, adenovirus 5-hACE2 mice $^{31}$ and ferrets, that can be used for immune response studies and preclinical evaluation of vaccines and therapeutics. In the clinic, Chinese immunologists detailed the nature of $\mathrm{T}$ cell and antibody responses to SARS-CoV-2 $\left(\mathrm{REFS}^{32-34}\right)$, identified the kidney as one of the main targets of SARS-CoV-2 infection ${ }^{35}$, described the virus-associated cytokine storm $^{36}$ and promoted clinical use of thymosin $^{37}$ and anti-IL- 6 therapies ${ }^{38}$ for patients with severe disease. Next, the Chinese Society for Immunology will propose national major research projects to further our insight into the immune mechanisms involved in coronavirus infections and to develop innovative technologies to tackle these emerging infectious diseases. Just as before, the Chinese Society for Immunology is fully committed to enhancing cooperation with IUIS and all regional societies to re-energize global scientific movements.

\section{Cuban Society for Immunology and Latin American and Caribbean Immunology Association: Ana B. Perez and Oliver Pérez. The Cuban Society for} Immunology has more than 1,000 associated (106 titular) members, who are active in basic or clinical immunology research and work in the public health system, scientific institutions and universities and also with the Latin American and Caribbean Immunology Association (La Asociación Latinoamericana y Caribeña de Inmunología) and the IUIS. As prominent members of the Cuban scientific community, our immunologists were called on to respond to the challenges of the COVID-19 pandemic. As early as 12 February 2020, a month before the first case was detected in Cuba (11 March), the Cuban government approved the creation of the Cuban Science Group for the Confrontation of COVID-19, which was key for decision-making in order to better control the predicted epidemic ${ }^{39,40}$.

As a result of this coordinated work, several immunological approaches were approved and applied during the Cuban COVID-19 outbreak. First, an antibody screening test was developed and applied in Cuba. Second, patients with COVID-19 were treated with immunotherapeutics developed in Cuba, including human recombinant interferon alfa- $2 b^{41}$, later combined with interferon- $\gamma$, a humanized recombinant monoclonal antibody to CD6 (REF. ${ }^{42}$ ) and an immunomodulatory peptide, CIGB-258, which is derived from heat shock protein 60 $(\text { HSP60 })^{43}$. Third, preventive strategies were introduced for vulnerable populations that used Cuban products to stimulate trained immunity or immunocompetence (Biomodulin T) ${ }^{40}$. Fourth, passive immunization with convalescent serum was used to treat critically ill patients, and mesenchymal stem cell therapy was used in patients with lung complications. Fifth, immunologists have identified biomarkers linked to disease severity and are trying to recognize correlates of protection. Sixth, immunologists with molecular biologists were involved in the development of vaccine candidates.

Each of these strategies has been equally and freely available to all of the Cuban population through a national integrated programme. Our members have been actively communicating the evolving science of COVID-19 through the national mass media, thereby contributing to furthering the public understanding of immunology. Moreover, Cuban medical and scientific experience has also contributed to the responses in many other countries during the COVID-19 pandemic.

At the time of writing, Cuba has 2,432 confirmed cases of and 87 deaths from COVID-19, with a recovery rate of $93.4 \%$. This relative success has been achieved due to Cuba's high physician-to-inhabitant ratio (1:116), an effective track and trace programme, the hospitalization of all people who tested positive and application of approved experimental treatments, which have helped to reduce morbidity and mortality associated with COVID-19.

\section{German Society for Immunology: Thomas Kamradt and Christine Falk.} During the pandemic, the German Society for Immunology (Deutsche Gesellschaft für Immunologie (DGfI)) has been supporting our members by participating in a number of educational and outreach efforts. For example, our members have contributed to webinars organized by the European Federation of Immunological Societies (EFIS) and the IUIS. The DGfI has also been advising our national politicians 
during the pandemic. For example, we were asked to advise the National Ethics Council (Deutscher Ethikrat) on the feasibility and usability of so-called immunity passports for people who have recovered from COVID-19. Moreover, the DGfI and the German Society for Virology have jointly advised the regulatory authorities on important safety issues related to the development and testing of vaccines against SARS-CoV-2.

Going forwards, we think immunology societies can have an important role in public engagement. The DGfI certainly appreciates that there is a lack of understanding of basic immunological concepts in large segments of the general population. We need to increase our efforts in public outreach far beyond the current outbreak. The DGfI has a standing committee on public outreach and has produced an information booklet entitled "Immunology for Everyone".

We encourage all immunologists to join their local immunology society if possible. The benefits of joining include first-class educational offers, networking opportunities, professional growth and the opportunity to become involved in public outreach. Promoting equality and diversity is another major strategic aim of the DGfI. This is reflected, for instance, in the fact that $50 \%$ of the members of the DGfI's governing council are women, a large proportion of invited speakers at the annual meetings of our society are women and childcare support is offered at our annual meetings.

Finally, we acknowledge the good work immunology societies can do in promoting international collaboration. At the beginning of the pandemic, the DGfI received a highly encouraging letter of solidarity from the Japanese Society for Immunology (JSI), in which it offered enormously generous practical help (for instance, in importing and maintaining critical strains of mice for facilities that had to be closed down).

\section{Israeli Immunological Society: Mira Barda-Saad and Amiram Ariel. When} the COVID-19 pandemic reached Israel in early 2020, a firm and swift government response elicited citizen adherence with social distancing instructions, containing the disease and preventing a more serious outbreak. As of 22 June, 20,778 people in Israel had been infected with SARS-CoV-2, with 40 patients still in a critical condition and 306 deaths. The immunological community rapidly responded to assist in various ways, including through the formation of multidisciplinary discussion forums, boosting research on the virus and supplementing the Ministry of Health's COVID-19 testing facilities with advanced PCR equipment and trained personnel. Special funding programmes were created by the Israel Science Foundation, the Israel Innovation Authority and the Ministry of Health with an expedited review process to explore various aspects of the pandemic. These included a variety of immunological research programmes ranging from innovative vaccination strategies to immune therapy, and strategies for inhibition of the cytokine storm induced by the virus. Israeli Immunological Society members provided support in refereeing these applications, serving on COVID-19-related policy-setting committees and performing research to identify therapeutic targets and immunogenic epitopes for vaccines. Several advanced vaccination development programmes are under way at the Israel Institute for Biological Research, academic institutes, medical centres and biotech companies.

The Israeli Immunological Society shared its communication resources and databases to promote these efforts and to assist in rapid and responsible containment of COVID-19 in Israel. We expect that the outstanding multidisciplinary efforts and innovative approaches that arose in these challenging times will remain as a future platform for biomedical research teams even after COVID-19 is under control.

\section{Italian Society of Immunology, Clinical Immunology and Allergology: Angela Santoni, Francesco Annunziato and}

Marco A. Cassatella. Italy was the first European country to face the outbreak of COVID-19 and suffered one of the highest death tolls in the world, leading the Italian government to rapidly take very restrictive measures. Italian Society of Immunology, Clinical Immunology and Allergology (SIICA) immunologists have been at the forefront of the Italian response to the COVID-19 pandemic, being interviewed by the most relevant TV programmes and journalists, and our members have worked hard to ensure rigorous dissemination of the science. This is particularly important in these 'infodemic' times. In addition, Alberto Mantovani and Guido Forni, who are both prominent SIICA immunologists and members of the Italian Science Academy (Accademia Nazionale delle Scienze), have written a document on different aspects of the COVID-19 pandemic, including issues related to the production of a vaccine ${ }^{44}$.

It has been challenging for SIICA to maintain its usual level of activities during these times of social distancing. However, our society supported several educational events on SARS-CoV-2 and COVID-19 between March and June 2020, and we hosted a 'School of Immunology' on the topic of viral immunology and vaccinology. This was organized by the SIICA junior faculty and had more than 400 students actively participating in interactive live webinars and scientific games. To mark the occasion of the 'Day of Immunology' on 29 April - which was this year dedicated to autoimmunity and autoinflammation SIICA released the 'Pills of Immunology' on its official YouTube channel, where several SIICA scientists discussed how the immune system is involved in fighting infections and chronic diseases, including COVID-19 $\left(\mathrm{REF}^{45}\right)$. Finally, SIICA and other European immunology societies strongly endorsed the GAVI advanced market commitment for COVID-19 vaccines (COVAX AMC), which is an initiative to support the development of a COVID-19 vaccine through multilateral, international collaborations ${ }^{46}$. Additional initiatives on COVID-19 education for the Italian public are already scheduled for the autumn, testifying to how SIICA is working hard to correctly inform public opinions surrounding the pandemic and vaccines.

\section{Japanese Society for Immunology:} Hiroshi Kiyono. At the time of writing (early July 2020), the spread of SARS-CoV-2 in Japan has fortunately been controlled, although occasional increases of new cases are seen in large cities, and most Japanese laboratories have been able to resume their research activities under restricted regulations. However, the pandemic continues worldwide, and the JSI wishes to extend its support and sincere appreciation to fellow physicians and medical staff across the world fighting on the front line against COVID-19, as well as to friends and colleagues all over the world who are suffering from the lockdown. The JSI believes that the solidarity of scientists will help and lead to the medical solutions and many of the social solutions that are necessary to deal with the COVID-19 pandemic. To this end, we deliver a message of solidarity and support to all regional immunology societies, expressing our firm intention to help each other overcome the pandemic together ${ }^{47}$

Going forwards, the fundamental mission of our society is to support our scientists in their attempts to unravel the immunological mechanisms by which the host responds to SARS-CoV-2 and to understand why some patients with 
COVID-19, but not others, develop severe disease. For this purpose, we believe that we should support both basic and clinical researchers with backgrounds in virology, immunology and infectious diseases, as well as other scientific fields, and bring these researchers together as one team. The JSI intends to play a key role in this through the creation of a scientifically interactive platform that will help us to understand the host immune response to SARS-CoV-2. To start this mission, we are now in the process of organizing an online international symposium focusing on the immune system in COVID-19, which will be held in December 2020 as the 49th annual meeting of the JSI ${ }^{48}$. Furthermore, JSI members are providing their scientific resources and knowledge for the development of vaccines and immunotherapies for COVID-19 in response to projects initiated by the Japanese Agency for Medical Research and Development (AMED) ${ }^{49}$.

The JSI believes that by showing a spirit of global solidarity and coming together as 'Team Immunology', our immunology communities can help to achieve our common goal of eradicating SARS-CoV-2.

\section{Russian Scientific Society of Immunologists: Valeriy A. Chereshnev.} Russia prepared thoroughly for the COVID-19 pandemic. The country's leadership, in cooperation with the scientific community, quickly introduced an isolation regime, calling on the medical community and citizens to help fight the pandemic. The Russian Scientific Society of Immunologists, the Russian Academy of Sciences, the Ministry of Health, and several other departments organized online conferences, at which specialists discussed various issues related to SARS-CoV-2. In educational programmes, immunologists and virologists covered various aspects of the pandemic, ranging from the dynamics of the immune response to treatment regimens and the creation of vaccines. Today, immunology has reached the very cutting edge of the fight against the pandemic and, as they say, 'we have all become immunologists and virologists. Therefore, immunologists were invited to the presidential commission to combat COVID-19.

In June 2020, a clinical trial of the first Russian vaccine against COVID-19 was started, and several more vaccine variants are on the way. Assessing the preliminary results of the fight against SARS-CoV-2, we come to the following conclusions. First, that quick, decisive adoption of public health measures, based on the opinion of specialists, is the main weapon in the fight against this infection. Second, that new schemes for the provision of medical care and social services must be implemented, with the understanding that it is the behaviour of citizens that determines the trajectory of a pandemic. Third, the best ideas of the theory and practice of immunology, virology and epidemiology should become the basis for all decision-making in order to combat the pandemic. Going forwards, the Russian Scientific Society of Immunologists will be involved in supporting all of these aims.

\section{Senegalese Society of Immunology: Alioune Dieye, Moustapha Mbow, Babacar Mbengue and Maguette D. S.}

Niang. Through its members, the Senegalese Society of Immunology (SSI) has joined the task force of the Ministry of Health and Social Action (Ministère de la Santé et de l'Action sociale du Sénégal) and the COVID-19 observatory of the Ministry of Higher Education, Research and Innovation (Ministère de l'Enseignement supérieur, de la Recherche et de l'Innovation) in Senegal. As part of such bodies, the SSI provides advice on the diagnostic methods for SARS-CoV-2 and has made proposals to the Ministry of Health and Social Action on the roles of laboratories and the contribution of immunology research to the COVID-19 pandemic. Within this framework, an algorithm for the use of SARS-CoV-2 antibodies has been developed and presented to the Ministry of Health and Social Action, and an overview of global research efforts, including on social science aspects of the pandemic, has been presented to the Ministry of Higher Education, Research and Innovation.

The SSI is also conducting research projects focused on (1) the immunological mechanisms underlying the clinical outcome of patients infected with SARS-CoV-2, (2) the immune correlates associated with protection from or susceptibility to COVID-19, with particular focus on populations from urban and rural regions and on any differences seen between Africans and Europeans, and (3) determining the existence and longevity of cellular and humoral memory responses to SARS-CoV-2.

Although action regarding social equality has not yet been considered in our response to the pandemic, investigation of discrimination will be taken into account. To ensure that our COVID-19-related activities help to further popularize immunology at the community level in
Senegal, we believe that the dissemination of our research findings should not only be limited to peer-review journals and scientific conferences but should also involve mainstream media outlets. We hope to hold future symposia where medical staff can stimulate interest in the importance of immunology and of joining immunology societies.

\section{South African Immunology Society:} Melinda Suchard. At the time of writing, South Africa is the African country most severely affected by COVID-19 in terms of the total numbers of confirmed cases and deaths. The expertise developed in other infectious diseases facilitated a rapid and sustained public health response. Members of the South African Immunology Society are involved in every aspect of the public health response, including molecular testing, evaluation of serology, clinical care, vaccine and treatment trials, and policy development. The society has enabled rapid sharing of information across disciplines and institutions through our well-received newsletters and a planned set of webinars.

COVID-19 has starkly highlighted the fundamental role of immunology for public health action, and we see an increasing trend of public users on our social media platforms seeking to understand the science behind the pandemic response strategies. The keen public interest in COVID-19 vaccine development is a tool to be leveraged to strengthen vaccine acceptance more generally among the social media-savvy generation.

The pandemic has brought diverse sectors together in solidarity, a noticeable trend within various medical disciplines that all seek deeper insights into the pathogenesis of COVID-19. A research focus for the country will undoubtedly include studies addressing the interaction of COVID-19 with other infectious diseases, particularly tuberculosis and HIV infection, as well as with non-communicable diseases, including hypertension and diabetes, which are highly prevalent in the country.

We anticipate a growing member base of South African and international society members as we work towards hosting the next meeting of the IUIS Congress in Cape Town in 2022.

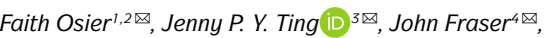
Bart N. Lambrecht ${ }^{5 凶}$, Marta Romano ${ }^{6}$, Ricardo T. Gazzinelli ${ }^{7 凶}$, Karina R. Bortoluci iD ${ }^{8}$ Dario S. Zamboni (iD ${ }^{9}$, Arne N. Akbar iD ${ }^{10 凶}$, Jennie Evans ${ }^{11}$, Doug E. Brown ${ }^{11}$, Kamala D. Patel ${ }^{12,13 凶}$ Yuzhang Wu ${ }^{14 凶}$, Ana B. Perez ${ }^{15 凶}$, Oliver Pérez ${ }^{16}$, Thomas Kamradt ${ }^{17 凶}$, Christine Falk ${ }^{18}$, Mira Barda-Saad ${ }^{19}$, Amiram Ariel (iD ${ }^{20}$,
} 
Angela Santoni2 ${ }^{21,22 凶}$, Francesco Annunziato ${ }^{23,24}$, Marco A. Cassatella ${ }^{25}$, Hiroshi Kiyono iD 26,27,28凶, Valeriy Chereshnev ${ }^{29 凶}$, Alioune Dieye ${ }^{30 凶}$, Moustapha Mbow ${ }^{30}$, Babacar Mbengue ${ }^{30}$, Maguette D. S. Niang iD ${ }^{30}$ and Melinda Suchard (DD ${ }^{31,32 凶}$ 'Biosciences Department, KEMRI Wellcome, Kilifi, Kenya. ${ }^{2}$ Parasitology Department, Heidelberg University Hospital, Heidelberg, Germany.

${ }^{3}$ Department of Genetics, Lineberger Comprehensive Cancer Center, University of North Carolina Chapel Hill, Chapel Hill, NC, USA.

${ }^{4}$ Faculty of Medical and Health Sciences, University of Auckland, Auckland, New Zealand.

${ }^{5}$ VIB-UGent Center for Inflammation Research, Ghent, Belgium

${ }^{6}$ Sciensano, Brussels, Belgium

${ }^{7}$ Fundação Oswaldo Cruz, Rio de Janeiro, Brazil. ${ }^{8}$ Universidade Federal de São Paulo, São Paulo, Brazil. ${ }^{9}$ Faculdade de Medicina de Ribeirão Preto, Universidade de São Paulo, São Paulo, Brazil.

${ }^{10}$ Division of Infection and Immunity, University College London, London, UK.

${ }^{11}$ British Society for Immunology, London, UK. ${ }^{12}$ Department of Physiology and Pharmacology, University of Calgary, Calgary, $A B$, Canada.

${ }^{13}$ Department of Biochemistry and Molecular Biology, University of Calgary, Calgary, $A B$, Canada.

${ }^{14}$ Institute of Immunology, Army Medical University, Chongqing, China.

${ }^{15}$ Pedro Kourí Institute, Havana, Cuba.

${ }^{16}$ Instituto de Ciencias Básicas y Preclínicas 'Victoria de Girón', Universidad de Ciencias Médicas de la Habana, Havana, Cuba

\section{${ }^{17}$ Institute of Immunology, Jena, Germany.}

${ }^{18}$ DZIF, Hannover Medical School, Hannover, Germany.

${ }^{19}$ Laboratory of Molecular and Applied Immunology, The Mina and Everard Goodman Faculty of Life Sciences, Bar-llan University, Ramat Gan, Israel. ${ }^{20}$ Department of Human Biology, Faculty of Natural Sciences, University of Haifa, Haifa, Israel.

${ }^{21}$ Department of Molecular Medicine, Sapienza University of Rome, Rome, Italy.

${ }^{22}$ Instituto Pasteur Italia, Cenci Bolognetti Foundation, Rome, Italy.

${ }^{23}$ School of Medicine, University of Florence,

Florence, Italy.

${ }^{24}$ Flow Cytometry Diagnostic Core, Careggi University Hospital, Florence, Italy.

${ }^{25}$ University of Verona, Verona, Italy.

${ }^{26}$ Division of Mucosal Immunology, Institute of Medical Science, University of Tokyo, Tokyo, Japan.

${ }^{27}$ Institute for Global Prominent Research, Graduate School of Medicine, Chiba University, Chiba, Japan.

${ }^{28}$ Center for Mucosal Immunology, Allergy and Vaccines, Division of Gastroenterology, Department of Medicine, School of Medicine, University of California, San Diego, San Diego, CA, USA.

${ }^{29}$ Institute of Immunology and Physiology, Ural Branch of Russian Academy of Sciences, Yekaterinburg, Russia.

${ }^{30}$ Department of Immunology, Faculty of Medicine, Pharmacy and Odontology, Cheikh Anta Diop University of Dakar, Dakar, Senegal.

${ }^{31}$ National Institute for Communicable Diseases, a division of the National Health Laboratory Service, Johannesburg, South Africa.
${ }^{32}$ Faculty of Health Sciences, University of the Witwatersrand, Johannesburg, South Africa.

凶e-mail: fosier@kemri-wellcome.org; jenny_ting@ med.unc.edu; j.fraser@auckland.ac.nz; bart.lambrecht@ ugent.be; ricardo.gazzinelli@fiocruz.br; a.akbar@ ucl.ac.uk; kpatel@ucalgary.ca; wuyuzhang@tmmu. edu.cn; anab@ipk.sld.cu; thomas.kamradt@med. uni-jena.de; mira.barda-saad@biu.ac.il; angela. santoni@uniroma1.it; kiyono@ims.u-tokyo.ac.jp; mchereshneva@mail.ru; alioune.dieye@ucad.edu.sn; melindas@nicd.ac.za

https://doi.org/10.1038/s41577-020-00428-4

Published online 10 September 2020

1. International Union of Immunological Societies. IUIS-Frontiers webinar series on COVID-19. IUIS https://iuis.org/webinars/ (2020).

2. The American Association of Immunologists. The American Association of Immunologists, public affairs. AAI https://www.aai.org/Public-Affairs (2020).

3. The American Association of Immunologists. Letter from the American Association of Immunologists. AAI https://www.aai.org/AAISite/media/Public_Affairs/ Letters-Comments/Letter-from-The-American-Associat ion-of-Immunologists-re-COVID-19.pdf (2020).

4. The American Association of Immunologists. Letter to $\mathrm{Dr}$ Collins regarding grant termination. AAl https://www.aai.org/AAISite/media/Public_Affairs/ Letters-Comments/Letter to Dr Collins Re Grant Termination_052020.pdf (2020).

5. The American Association of Immunologists. Statement of AAI Committee on Public Affairs. AAI https://www.aai.org/AAISite/media/Public Affairs/ Letters-Comments/AAI-Opposes-US-Withdrawal-fromWHO-and-Visa-Changes-Affecting-Chinese-Scientists060920.pdf (2020)

6. The Journal of Immunology COVID-19, SARS, and MERS articles. J. Immunol. https://www.jimmunol.org/ coronaviruses

7. The Journal of Immunology. A message to our authors, reviewers, and readers. J. Immunol. https://www.jimmunol.org/content/messageour-authors-reviewers-and-readers7 (2020).

8. The American Association of Immunologists. Poster abstracts from IMMUNOLOGY2020. AAI https://plan. core-apps.com/aai2020/abstracts (2020).

9. The American Association of Immunologists COVID-19 resources and information center. $A A I$ https:/www.aai.org/COVID-19-Resources (2020).

10. The American Association of Immunologists. AAI Courses in immunology. AAl https://www.aai.org/ Education/Courses (2020).

11. The American Association of Immunologists. AAI Fellowships. $A A$ l https://www.aai.org/Careers/ Fellowships/ (2020)

12. The Australian and New Zealand Society for Immunology. ASI community. ASI https:// www.immunology.org.au/community/ (2020).

13. The Australian and New Zealand Society for Immunology. ASI Fireside chats. ASI https:// www.immunology.org.au/events/list/ (2020).

14. Bosteels, C. et al. Sargramostim to treat patients with acute hypoxic respiratory failure due to COVID-19 (SARPAC): A structured summary of a study protocol for a randomised controlled trial. Trials 21, 491 (2020).

15. Maes, B. et al. Treatment of severely ill COVID-19 patients with anti-interleukin drugs (COV-AID): a structured summary of a study protocol for a randomised controlled trial. Trials 21, 468 (2020).

16. US National Library of Medicine. ClinicalTrials.gov https://www.clinicaltrials.gov/ct2/show/NCT0 4382755 (2020).

17. Sociedade Brasiliera de Imunologia. SBI homepage. SBI https://sbi.org.br/ (2020).

18. British Society for Immunology. Information on the Joint Academy of Medical Sciences/British Society for Immunology Taskforce: https://www.immunology.org/ coronavirus/immunology-and-covid-19 (2020).

19. British Society for Immunology. Joint Academy of Medical Sciences/British Society for Immunology report on COVID-19 and immunology https://www. immunology.org/sites/default/files/Final_COVID-19. Immunology_report.pdf (2020).

20. British Society for Immunology. Coronavirus information hub on the BSI website. BSI https://www.immunology.org/coronavirus (2020).

21. British Society for Immunology. BSI 'Connect on Coronavirus' webinar series. BSI https://www.immunology.org/coronavirus/ connect-coronavirus-webinars (2020).

22. Canadian Society for Immunology. CSI Council statement on systemic inequities. CSI https://www. csi-sci.ca/ (2020).

23. Cai, X. F. et al. A peptide-based magnetic chemiluminescence enzyme immunoassay for serological diagnosis of coronavirus disease 2019. J. Infect. Dis. 222, 189-193 (2020).

24. Diao, B. et al. Diagnosis of acute respiratory syndrome coronavirus 2 infection by detection of nucleocapsid protein. Preprint at medRxiv https:// doi.org/10.1101/2020.03.07.20032524 (2020).

25. Gao, Q. et al. Rapid development of an inactivated vaccine candidate for SARS-CoV-2. Science https:// doi.org/10.1126/science.abc1932 (2020).

26. Zhu, F. C. et al. Safety, tolerability, and immunogenicity of a recombinant adenovirus type- 5 vectored COVID-19 vaccine: a dose-escalation, open-label, non-randomised, first-in-human trial. Lancet 395 1845-1854 (2020).

27. Chen, $X$ Y et al. Human monoclonal antibodies block the binding of SARS-CoV-2 spike protein to angiotensin converting enzyme 2 receptor. Cell. Mol. Immunol. 17, 647-649 (2020).

28. Cao, Y. et al. Potent neutralizing antibodies against SARS-CoV-2 identified by high-throughput single-cell sequencing of convalescent patients' B cells. Cell https://doi.org/10.1016/j.cell.2020.05.025 (2020).

29. Wu, Y. et al. A noncompeting pair of human neutralizing antibodies block COVID-19 virus binding to its receptor ACE2. Science 368, 1274-1278 (2020).

30. Bao, L. et al. The pathogenicity of SARS-CoV-2 in hACE2 transgenic mice. Nature https://doi.org/ 10.1016/10.1038/s41586-020-2312-y (2020).

31. Sun, J. et al. Generation of a broadly useful model for COVID-19 pathogenesis, vaccination, and treatment. Cell https://doi.org/10.1016/j.cell.2020.06.010 (2020).

32. Diao, B. et al. Reduction and functional exhaustion of T cells in patients with coronavirus disease 2019 (COVID-19). Front. Immunol. 11, 827 (2020).

33. Long, Q. X. et al. Antibody responses to SARS-CoV-2 in patients with COVID-19. Nat. Med. 26, 845-848 (2020).

34. Long, Q. X. et al. Clinical and immunological assessment of asymptomatic SARS-CoV-2 infections. Nat. Med. https://doi.org/10.1038/ s41591-020-0965-6 (2020).

35. Diao, B. et al. Human kidney is a target for novel severe acute respiratory syndrome coronavirus 2 (SARS-CoV-2) infection. Preprint at medRxiv https:/ doi.org/10.1101/2020.03.04.20031120 (2020).

36. Zhou, Y. et al. Pathogenic T-cells and inflammatory monocytes incite inflammatory storms in severe COVID-19 patients. Natl Sci. Rev. 7, 998-1002 (2020).

37. Liu, Y. et al. Thymosin alpha 1 (Ta1) reduces the mortality of severe COVID-19 by restoration of lymphocytopenia and reversion of exhausted T cells. Clin. Infect. Dis. https://doi.org/10.1093/cid/ciaa630 (2020).

38. Xu, X. et al. Effective treatment of severe COVID-19 patients with tocilizumab. Proc. Natl Acad. Sci. USA 117, 10970-10975 (2020)

39. Díaz-Canel Bermúdez, M., Núñez Jover, J. Government management and Cuban science in the confrontation with COVID-19. An. Acad. Cienc. Cuba. http://www. revistaccuba.cu/index . php/revacc/article/view/881/892 (2020).

40. Castellanos-Serra, L. Bringing Cuban Biotech Research to Bear on COVID-19: All Hands and Minds on Deck. MEDICC Rev. 22, 31-37 (2020).

41. Pereda, R., et al. Therapeutic effectiveness of interferon-alpha2b against COVID-19: the Cuban experience. Preprint at medRxiv https:// doi.org/10.1101/2020.05.29.20109199 (2020).

42. Saavedra, D., et al. An anti-CD6 monoclonal antibody (itolizumab) reduces circulating IL-6 in severe covid-19 elderly patients. Immun. Ageing https://doi.org/10.21203/rs.3.rs-32335/v1 (2020)

43. Venegas-Rodríguez, R., et al. CIGB-258 immunomodulatory peptide: a novel promising treatment for critical and severe COVID-19 patients. Preprint at medRxiv https://doi.org/10.1101/ 2020.05.27.20110601 (2020).

44. Accademia Nazionale dei Lincei. COVID19. An executive report. https://www.lincei.it/it/article/ covid-19-executive-report (2020).

45. Società Italiana dilmmunologia, Immunologia Clinica e Allergologia. SIICA SCHOOL, official channel. Youtube https://www.youtube.com/channel UCh5jjFh6tOARju4IKIvUHKO (2020). 


\section{VIEWPOINT}

46. Gavi, the Vaccine Alliance. The GAVI COVAX AMC Gavi https://www. gavi.org/sites/default/files/2020-06/ Gavi-COVAX-AMC-IO.pdf (2020).

47. The Japanese Society of Immunology. Letter of solidarity and support from the Japanese Society of Immunology. JSI http://files.jsi-men-eki.org general/meneki/JSI_Message 20200327.pd (2020).

48. The Japanese Society of Immunology. Homepage for the 49th Meeting of the Japanese Society for Immunology. JSI http://icongroup.co.jp/49immunology/ english/ (2020).

49. Japanese Agency for Medical Research and Development. AMED new scientific outputs related to COVID-19. AMED https://www.amed.go.jp/en/news topics/nso-index.html (2020).

\section{Acknowledgements}

F.O. acknowledges the members of the International Union of Immunological Societies (IUIS) Executive Committee and particularly the Secretary General for their contributions. She thanks the members of the publication committee led by R. Carsetti for their efforts and leadership in supporting the IUIS/Frontiers scientific webinars. She also thanks Frontiers for its continued partnership with the IUIS, which has been instrumental to the success of the webinars. A.D. M.M. B.M. and M.D.S.N. thank the Senegalese Ministry of Health and Social Action and Ministry of High Education, Research and Innovation for their support. The British Society for Immunology (BSI) coronavirus work is supported by the Lorna and Yuti Chernajovsky Biomedical Research Foundation, with additional support from the following BSI Gold Corporate Members: 10x Genomics, Fluidigm, Miltenyi Biotec and NanoString. H.K. acknowledges the great contribution of the Japanese Society for Immunology (JSI) executive board members K. Takeda (Osaka University), K. Hase (Keio University), O. Takeuchi (Kyoto University) and A. Takaoka (Hokkaido University) for supporting JSI's emphasis on solidarity for scientists, and extends sincere appreciation to them on behal of all JSI members. M.S. thanks the following members of the South African Immunology Society Executive Committee for their input: E. Mayne (University of the Witwatersrand and National Health Laboratory Service), C. Gray (University of Cape Town and National Health Laboratory Service) K. Kgoadi (University of Cape Town), S. Buldeo (Neuberg Globa Laboratory), C. Worsley (University of the Witwatersrand), H. Ranchod (National Institute for Communicable Diseases), J. Peter (University of Cape Town), T. Scriba (University of
Cape Town) and R. Glashoff (Stellenbosch University). Nature Reviews Immunology apologizes to all of the regional and national immunology societies that we were not able to feature in this article for reasons of space restrictions. We encourage all of our readers to join and support the work of their relevant immunology societies. Finally, we thank all immunologists and immunology societies for their monumental contributions to tackling the COVID-19 pandemic.

Competing interests

J.P.YT is a co-founder of IMMvention Therapeutix and Goldcrest Bio. All other authors declare no competing interests.

Publisher's note

Springer Nature remains neutral with regard to jurisdictional claims in published maps and institutional affiliations.

\section{Supplementary information}

Supplementary information is available for this paper at https://doi.org/10.1038/s41577-020-00428-4.

(c) Springer Nature Limited 2020 